\title{
Evaluation of upper extremity reachable workspace using Kinect camera
}

\author{
Gregorij Kurillo $^{\mathrm{a}, \mathrm{b}, *}$, Alic Chen ${ }^{\mathrm{c}}$, Ruzena Bajcsy ${ }^{\mathrm{a}}$ and Jay J. Han ${ }^{\mathrm{b}}$ \\ ${ }^{a}$ Department of Electrical Engineering and Computer Science, University of California at Berkeley, \\ Berkeley, CA, USA \\ ${ }^{\mathrm{b}}$ Department of Physical Medicine and Rehabilitation, University of California at Davis Medical \\ Center, Sacramento, CA, USA \\ ${ }^{\mathrm{c}}$ Department of Mechanical Engineering, University of California at Berkeley, Berkeley, CA, USA
}

Received 26 September 2013

Accepted 27 October 2013

\begin{abstract}
.
BACKGROUND: In clinical evaluation of upper extremity, there is a lack of assessment methods that are quantitative, reliable, and informative of the overall functional capability of an individual.

OBJECTIVE: We present new methodology for the assessment of upper extremity impairments based on the concept of 3dimensional reachable workspace using Microsoft Kinect.

METHODS: We quantify the reachable workspace by the relative surface area representing the portion of the unit hemisphere that is covered by the hand movement. We examine accuracy of joint positions, joint angles, and reachable workspace computation between the Kinect and motion capture system.

RESULTS: The results of our analysis in 10 healthy subjects showed that the accuracy of the joint positions was within $66.3 \mathrm{~mm}$ for our experimental protocol. We found that the dynamic angle measurements had relatively large deviations (between $9^{\circ}$ to $28^{\circ}$ ). The acquired reachable workspace envelope showed high agreement between the two systems with high repeatability between trials (correlation coefficients between 0.86 and 0.93 ).

CONCLUSIONS: The findings indicate that the proposed Kinect-based 3D reachable workspace analysis provides sufficiently accurate and reliable results as compared to motion capture system. The proposed method could be promising for clinical evaluation of upper extremity in neurological or musculoskeletal conditions.
\end{abstract}

Keywords: Kinect, upper extremity, functional evaluation, reachable workspace

\section{Introduction}

There are many clinical outcome measures in use pertaining to the upper extremity, such as: manual and quantitative muscle test (MMT, QMT), range of motion (ROM) [1], standardized timed function tests (e.g. 9-hole peg test) [2], Jebsen-Taylor hand function test [3], Wolf Motor Function Test [4], Brooke test [5], Fugl-Meyer assessment, and many others that were developed to evaluate impairments in specific conditions (e.g., [6-9]). One of the most commonly used standard measures across multiple

\footnotetext{
${ }^{*}$ Corresponding author: Gregorij Kurillo, University of California Berkeley, 752 Sutardja Dai Hall \#1764, Berkeley, CA 94720-1764, USA. Tel.: +1 510642 7456; E-mail: gregorij@eecs.berkeley.edu.
} 
conditions is ROM. Traditional ROM goniometry assessment consists of moving the joint through its angular range in different body planes and measuring the joint limits using a protractor goniometer. The ROM measurement using this method can suffer from low accuracy and large inter- and intra-examiner variability as it depends considerably on the experience of the clinical evaluator [10]. Furthermore, ROM information is typically provided as a list of numerical joint angle limits, which makes comparison of data challenging and visualization of the overall functional performance of an individual difficult.

To address the lack of clinical tools in this area, we propose a new methodology for the assessment of upper limb function using the concept of reachable workspace. The reachable workspace is wellestablished in robotics to graphically represent the boundaries of the working volume of a robotic manipulator [11]. Among the first applications of workspace analysis in human was the ergonomic evaluation of an airplane pilot's workspace envelope reported in [12]. Other researchers have investigated the workspace envelope by analyzing kinematic models of the upper extremity with an individual's anthropometric data [13-15]. Analysis of the human workspace has also been presented in several works related to the design of robotic devices for human-machine interaction [16,17].

Klopčar and Lenarčič [18] were the first to investigate how the reachable workspace volume could be used to evaluate the functionality of the upper extremity based on an articulated kinematics model of the arm and standardized goniometric measurements.

Since the standardized goniometric measurements can be time and resource intensive efforts, we propose to evaluate the reachable workspace from a well-defined movement protocol captured via a depth-sensing camera, such as the Microsoft Kinect. Initially we developed the methodology using a marker-based stereo system and demonstrated its discriminative properties to characterize upper-limb dysfunction in patients with various neuromuscular conditions [19]. While the use of the early system was effective for functional workspace characterization, its implementation in an applied clinical setting was hampered by the cost of the stereo camera, extended preparation time, and burden on the patient since the system required application of markers.

Consumer depth-sensing cameras, such as Microsoft Kinect, have in the recent years gained significant traction in various applications outside gaming. Unlike the motion capture system that requires users to wear markers, the Kinect utilizes an infrared-based depth sensor and a color camera for relatively accurate real-time 3D scene capture with built-in skeletal tracking capabilities [20]. To date, Microsoft Kinect has been evaluated for several clinical applications including postural control assessment [21], inhome fall risk assessment [22], upper-extremity function quantification [23], physical rehabilitation [24, 25], sports [26], and exercise [27].

In this paper, we focus on the technical aspect and accuracy of proposed methodology for assessment of reachable workspace as a potential outcome measure for the upper extremity. We extend our initial presentation in [28] by providing additional details on the methods and more comprehensive analysis of the data, including statistical analyses of the joint positions and angle accuracy, validity and reliability of the reachable workspace, and statistical test for the effects of gender and hand dominancy.

\section{Methods}

\subsection{Experimental setup and calibration}

For the evaluation of the reachable workspace, we used Kinect for Windows camera (Microsoft, Redmond, WA) which is capable of capturing color and depth images with 30 frames per second (fps). The depth acquisition is based on active sensing of infrared pattern projected onto the scene, which provides 


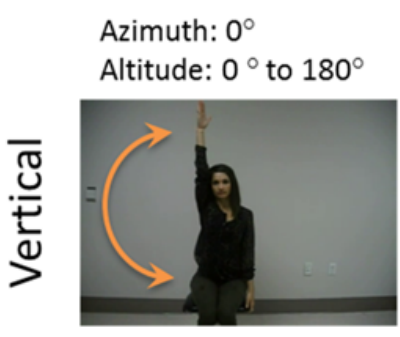

Azimuth: $0^{\circ}$ to $135^{\circ}$ Altitude: $30^{\circ}$

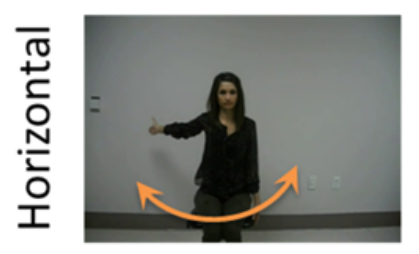

Azimuth: $45^{\circ}$

Altitude: $0^{\circ}$ to $180^{\circ}$

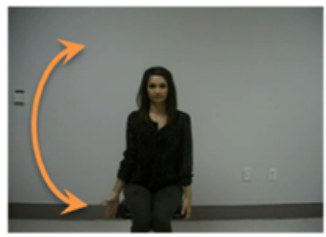

Azimuth: $0^{\circ}$ to $135^{\circ}$ Altitude: $90^{\circ}$

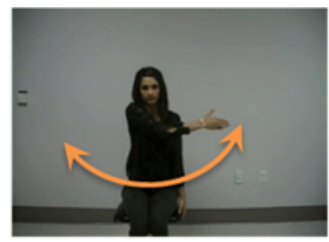

Azimuth: $90^{\circ}$

Altitude: $0^{\circ}$ to $180^{\circ}$

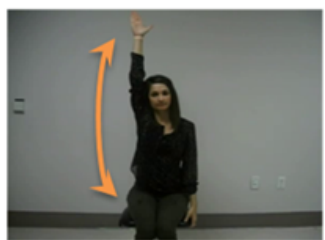

Azimuth: $0^{\circ}$ to $135^{\circ}$ Altitude: $0^{\circ}$ to $180^{\circ}$

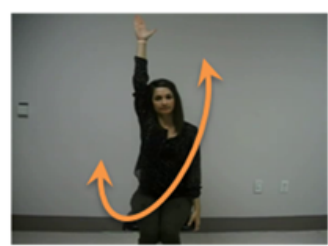

Azimuth: $135^{\circ}$

Altitude: $0^{\circ}$ to $180^{\circ}$

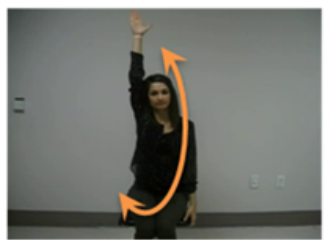

Azimuth: $90^{\circ}$ Altitude: $0^{\circ}$ to $-90^{\circ}$

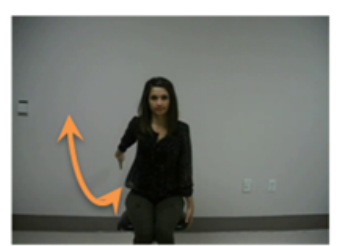

(a)

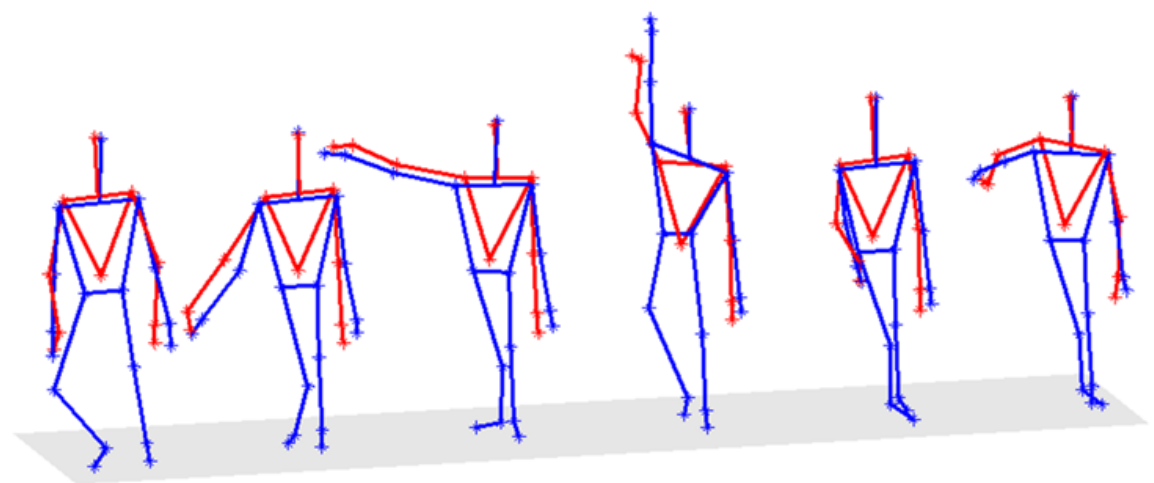

(b)

Fig. 1. (a) Key motions of the movement protocol for evaluation of the reachable workspace. (b) Comparison of Kinect (blue) and motion capture based skeletons (red) as captured in several key frames. (Colours are visible in the online version of the article; http://dx.doi.org/10.3233/THC-130764)

a robust estimation of depth at each pixel with the resolution of $320 \times 240$ pixels and accuracy of 10 $40 \mathrm{~mm}$ in the range of 1-4 $\mathrm{m}$ [29]. The skeletal tracking capability of the Microsoft Kinect SDK provides the 3D location of 20 joints of tracked user. The skeletal tracking is based on a per-pixel classification of the depth image into body parts from a large training data set, followed by fitting of a skeleton into the hypothesized joints with temporal continuity constraints [20].

As a reference, we simultaneously collected data with Impulse motion-capture system (PhaseSpace, Inc., San Leandro, CA), which can uniquely identify and track the 3D positions of LED markers with sub-millimeter accuracy at a frequency of $480 \mathrm{~Hz}$. For the validation experiments, each subject wore a tight-fitting shirt equipped with 18 markers on the upper body, 3 additional markers on dorsal side of each hand, and 3 markers on the head. In total, 3D positions of 27 markers were recorded. The location of the markers was chosen to correspond with the standard motion capture layout used for skeleton fitting where each body segment is outfitted with at least two markers. To analyze the motion of individual 

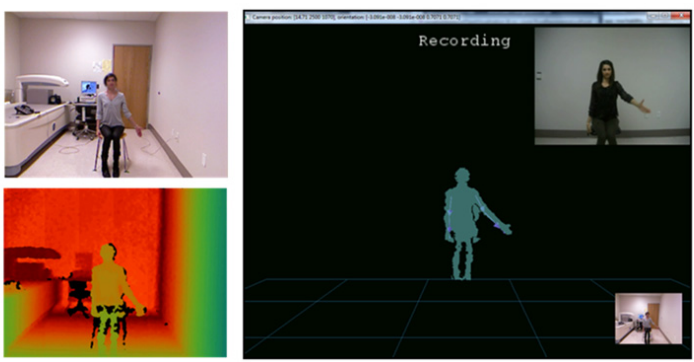

(a)

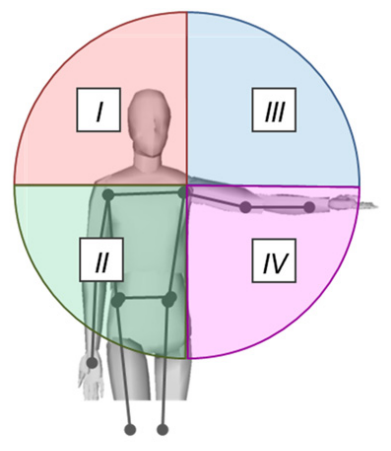

Left Side

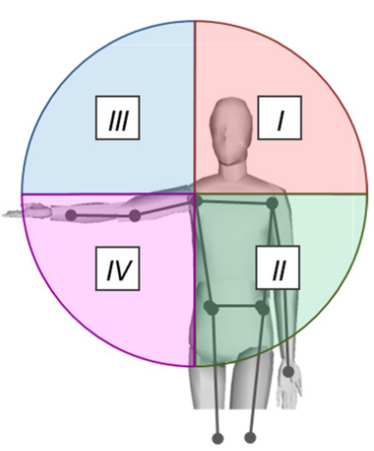

Right Side

(b)

Fig. 2. (a) Output color and depth data from the Kinect camera (left) and the corresponding visual feedback presented to subjects during the data acquisition (right). (b) Division of the reachable workspace into four quadrants for the left and right arm. (Colours are visible in the online version of the article; http://dx.doi.org/10.3233/THC-130764)

body segments, we rigged the markers with a skeleton using Recap 2.0 software (PhaseSpace, Inc.). Recap software includes a calibration procedure to determine the dimensions of the skeleton segments and the joint rotation axes from the movement of the surface markers. The calibration consists of rotating different body segments (e.g., wrist, elbow, shoulder) while standing in the standard T-pose. The final upper-body skeleton comprises of 17 joints which were matched with the upper-body kinematic structure of the Kinect by reducing the complexity of the spinal kinematic chain of the motion-capture system.

For data acquisition, the Kinect was positioned approximately $120 \mathrm{~cm}$ above the floor and centered under a 55" TV screen (used for feedback) with the motion capture cameras arranged in a circular form around the capture space. The subject was seated on a chair with a relatively small footprint to avoid interference and occlusion of body parts with data acquisition. The two systems were synchronized using a Network Time Protocol (NTP) service. To align the motion data in 3D space, the Kinect camera was first calibrated using the standard checkerboard method to obtain intrinsic parameters. In the second step, the projection transform between the Kinect color camera and motion capture was obtained from collected 3D point cloud generated by a moving LED marker. The alignment was further refined by estimating the rigid transformation between the skeleton joint trajectories of the two systems after the temporal alignment. Figure 1(b) shows the resulting skeletons after the geometric and temporal alignment.

\subsection{Upper extremity reachable workspace protocol}

The upper extremity movement protocol (Fig. 1(a)), initially presented in [19], was adapted for the use with the Kinect camera to cover cardinal movements of the shoulder. From the initial resting position of 
Table 1

Demographic and anthropometric data of the subjects

\begin{tabular}{|c|c|c|c|c|c|c|c|c|c|c|}
\hline \multirow[t]{2}{*}{ ID } & \multirow[t]{2}{*}{ Sex } & \multirow[t]{2}{*}{ Age } & \multirow{2}{*}{$\begin{array}{l}\text { Height } \\
(\mathrm{cm})\end{array}$} & \multirow{2}{*}{$\begin{array}{c}\text { Weight } \\
(\mathrm{kg})\end{array}$} & \multicolumn{3}{|c|}{ Dominant Side $\left({ }^{\circ}\right)$} & \multicolumn{3}{|c|}{ Non-dominant Side $\left({ }^{\circ}\right)$} \\
\hline & & & & & $\begin{array}{l}\text { Shoulder } \\
\text { flexion }\end{array}$ & $\begin{array}{l}\text { Shoulder } \\
\text { extension }\end{array}$ & $\begin{array}{c}\text { Shoulder } \\
\text { Abd. }\end{array}$ & $\begin{array}{l}\text { Shoulder } \\
\text { flexion }\end{array}$ & $\begin{array}{l}\text { Shoulder } \\
\text { extension }\end{array}$ & $\begin{array}{c}\text { Shoulder } \\
\text { Abd. }\end{array}$ \\
\hline 1 & $\mathrm{~F}$ & 29 & 166.0 & 53.2 & 171 & 67 & 180 & 178 & 55 & 182 \\
\hline 2 & $\mathrm{~F}$ & 15 & 162.5 & 62.7 & 170 & 66 & 181 & 166 & 57 & 172 \\
\hline 3 & $\mathrm{~F}$ & 54 & 162.0 & 68.0 & 152 & 47 & 156 & 147 & 44 & 150 \\
\hline 4 & $\mathrm{~F}$ & 16 & 162.5 & 52.3 & 168 & 70 & 177 & 174 & 51 & 177 \\
\hline 5 & $\mathrm{M}$ & 34 & 192.0 & 68.0 & 139 & 65 & 135 & 146 & 51 & 131 \\
\hline 6 & M & 41 & 178.0 & 75.0 & 142 & 54 & 150 & 141 & 56 & 148 \\
\hline 7 & $\mathrm{~F}$ & 32 & 154.0 & 48.0 & 175 & 63 & 186 & 170 & 55 & 183 \\
\hline 8 & M & 31 & 173.0 & 65.0 & 156 & 77 & 176 & 165 & 77 & 180 \\
\hline 9 & M & 26 & 173.0 & 62.0 & 164 & 62 & 166 & 157 & 66 & 164 \\
\hline 10 & M & 31 & 160.5 & 58.4 & 152 & 58 & 161 & 149 & 51 & 163 \\
\hline
\end{tabular}

adducted arm at the side of the body with the palm facing forward, the arm is lifted above the head while keeping the elbow extended, performing the same movements in the vertical planes of $0,45,90$, and 135 degrees. The second set of movements consisted of horizontal sweeps at the level of the umbilicus and shoulder. Both vertical and horizontal movements are performed in one recording session, lasting approximately 1 minute. To standardize the assessment and control for execution speed, the participants followed the movement through a video feedback. The feedback screen included a mirrored 3D image of the participant alongside a video playback of the movement protocol as shown in Fig. 2(a).

\subsection{Subjects and experimental procedure}

The reachable workspace evaluation was performed on 10 healthy subjects (mean age of females: $29.2 \pm 15.8$ years; mean age of males: $32.6 \pm 5.5$ years; combined mean age: $30.9 \pm 11.3$ years) who had no known history of upper extremity impairments and had a normal ROM in the shoulder. The demographic information and shoulder joint goniometry data, which was measured by a physical therapist, are reported in Table 1. In addition, we report on preliminary results obtained in a 74 year-old female subject with Facioscapulohumeral muscular dystrophy (FSHD), who was captured only by the Kinect camera at the clinic using the same movement protocol.

Each subject was first briefed on the experimental protocol and signed a consent form that was approved by the University Institutional Review Board Administration. After subject donned the suit with markers, we collected the skeleton calibration data. Next, the subject watched full-screen instructional video of the movement protocol. The kinesiologist provided additional instructions on body posture and limb positioning to ensure that no significant substitution or compensatory movements were utilized. Finally, three repetitions of the movement protocol on each side were recorded.

\subsection{Data analyses}

\subsubsection{Kinematic data processing}

The data collected from the motion-capture was first imported to Recap to generate the upper body skeleton based on the collected calibration data. For all recordings, occluded motion trajectories of markers were interpolated using cubic interpolation while the final trajectories of joints were filtered with an infinite impulse response (IIR) Butterworth filter with a cut-off frequency of $30 \mathrm{~Hz}$. The remainder of the analysis was performed in Matlab (MathWorks, Natick, MA), where the recordings from the motion 
capture and Kinect were aligned based on the time stamp data. The motion trajectories from the motioncapture system were re-sampled from $480 \mathrm{~Hz}$ to $30 \mathrm{~Hz}$ to match the temporal time stamps of the Kinect recordings.

\subsubsection{Joint estimation and angular analysis}

To analyze the robustness of the pose estimation of the Kinect skeleton tracking, we compared the location of the joints with those obtained from the motion-capture skeleton. For each measured skeleton pose $t$ and joint $j \in\{$ left shoulder, left elbow, left wrist, right shoulder, right shoulder, right wrist $\}$, we computed the Euclidean distance as: $D_{i, j}=\left\|x_{t, j, \text { Kinect }}-x_{t, j, \text { Mocap }}\right\|_{L 2}$. Due to differences in the kinematic structures of the two skeletons, the distance between the joint positions was not expected to be zero, but rather a constant value $\mu_{j}$ with some variance $\sigma_{j}$ around the joint location. The kinematic data was modeled by a simplified kinematic structure using a spherical joint in the shoulder and hinge joints in the elbow and the wrist. To further examine the variability of the angular measurements across all subjects and repetitions, we applied dynamic time warping (DTW) to angular measurements.

\subsubsection{Reachable workspace data analysis}

The key steps of the algorithm to determine the reachable workspace are presented in Fig. 3. The ideal reachable workspace for the given model is a spherical enclosure [14] encompassing the set of farthest points relative to the torso that an individual can reach naturally by moving their arm in full extension. To calculate the workspace boundaries, we first determined the parameters of the sphere by the least-squares fitting to the captured hand trajectory. Next, we transformed the trajectory coordinates into the spherical coordinates with the two angles corresponding to shoulder flexion/extension and abduction/adduction measurements. In the new parameterized space, we located the maximal boundaries of the trajectory by fitting a concave polygon to the data points using the alpha shape geometry with $\pi / 4$ radius [30]. The radius was chosen based on the approximate gap between the movement directions. To smooth the concave boundary, we applied Catmull-Rom splines [31] with the control points sampled from the original boundary polygon.

Next, we identified the points on the spherical surface that were located both inside and outside the boundary polygon, and back-projected that information into the Cartesians coordinates to cull the surface patches accordingly. Furthermore, we divided the workspace into four quadrants that corresponded to clinically relevant functional subspaces (e.g. above/below shoulder, ipsilateral/contralateral side of the body with shoulder joint as the origin in the sagittal plane as shown in Fig. 2(b)). The total surface area was determined as the sum of the active surface patches across the quadrants and was further normalized with respect to surface area of a unit hemi-sphere (by dividing it by a factor of $2 \pi R^{2}$ where $R$ corresponds to the radius of the fitted sphere) to allow comparison of the results between subjects. The assessed relative surface area (RSA) therefore lies between 0.0 and 1.0, where 1.0 represents reachable workspace envelope of the entire (frontal) hemi-sphere.

\subsection{Statistical analyses}

The validity of the reachable workspace evaluation was defined as the agreement of the Kinect-based measurement with the more accurate motion-capture system. The reliability of the assessment was defined as the consistency of the measure with repeated observations by the two systems. To assess the reliability, we analyzed three repeated measurements performed on the same day for the dominant and non-dominant arm. A one-way repeated-measure analysis of variance (ANOVA) was used to determine the reliability during each activity. Individual error scores of zero indicate reliability. Bland-Altman 


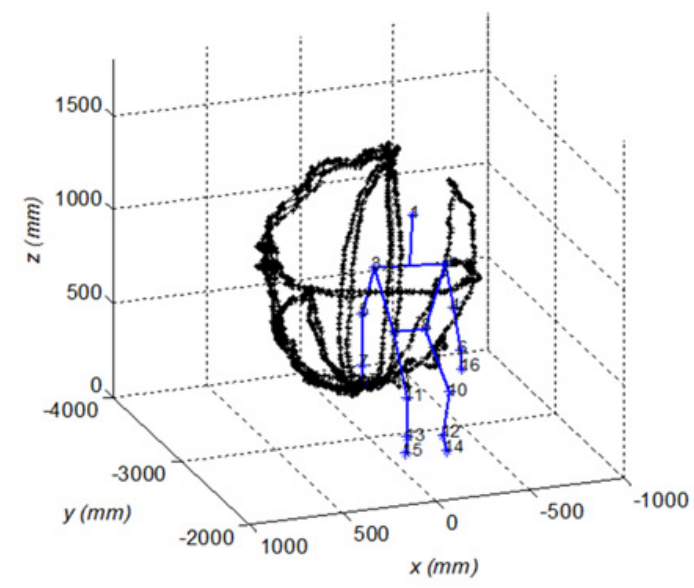

(a)

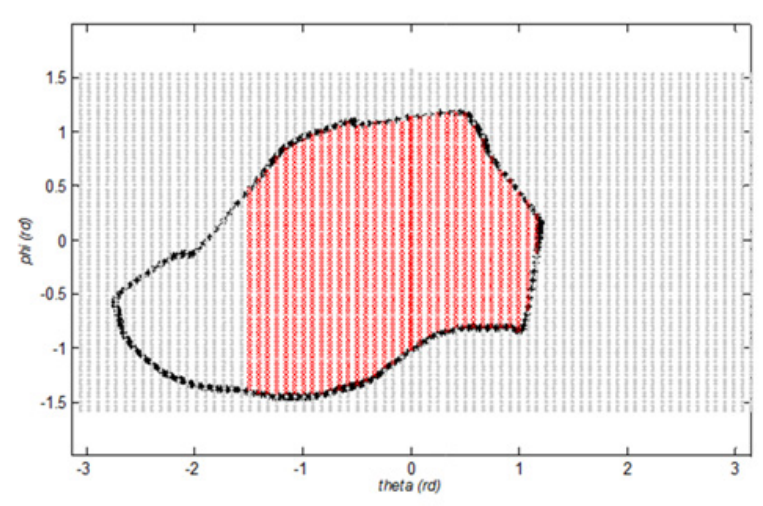

(c)

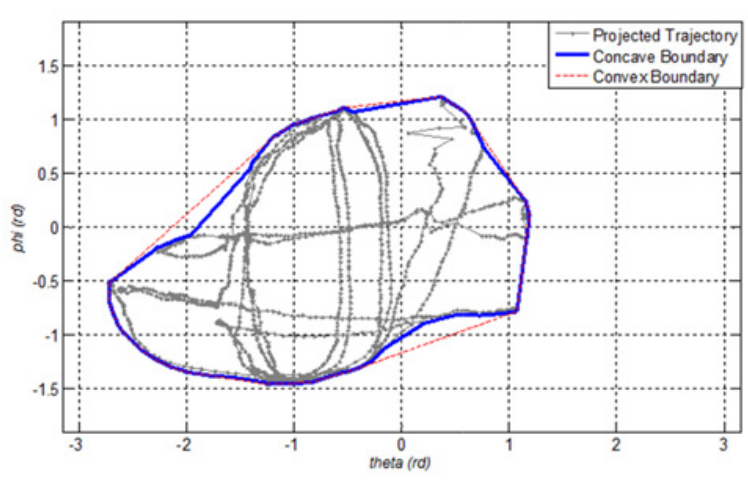

(b)

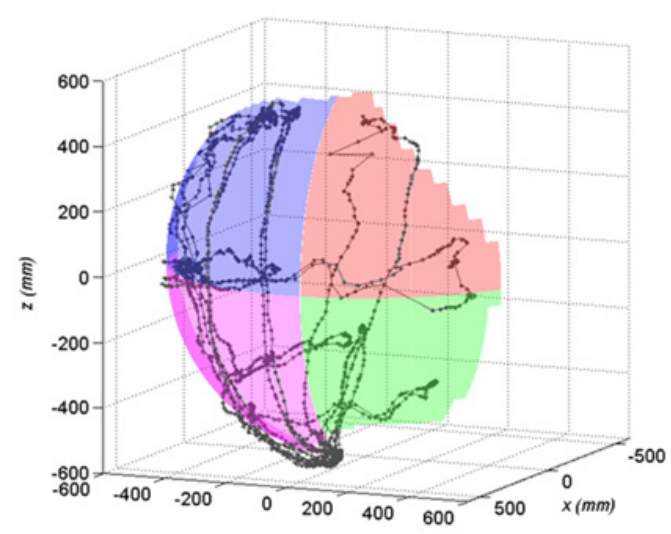

(d)

Fig. 3. Determination of the reachable workspace: (a) 3D trajectory is captured from the kinematics; (b) trajectory is parameterized and concave boundary is determined using alpha shapes; (c) concave boundary is used to determine the portion of the frontal hemisphere subject reached; (d) points on the sphere defined by the boundary are back-projected and divided into four quadrants. (Colours are visible in the online version of the article; http://dx.doi.org/10.3233/THC-130764)

plots were used to show the dispersion of the individual reliability of the measures. For the analysis of the average values of the parameters, we report arithmetic mean and the corresponding standard deviation intervals, unless otherwise noted. The statistical analysis was performed with Microsoft Excel and Matlab Statistical Toolbox.

\section{Results}

\subsection{Pose analysis}

To compare the Kinect system to the motion capture for the experimental protocol, measurement differences of the upper extremity joints were calculated and averaged for the 10 subjects. The offset of each joint is a time-averaged difference between the measurements from the two systems. The joint 
Table 2

Mean $(\mu)$ and standard deviation $(\sigma)$ joint position differences between Kinect and motion capture

\begin{tabular}{|c|c|c|c|c|}
\hline & \multicolumn{2}{|c|}{ Right side protocol } & \multicolumn{2}{|c|}{ Left side protocol } \\
\hline & $\mu(\mathrm{mm})$ & $\sigma(\mathrm{mm})$ & $\mu(\mathrm{mm})$ & $\sigma(\mathrm{mm})$ \\
\hline Right shoulder & 65.5 & 26.2 & 60.7 & 10.9 \\
\hline Right elbow & 57.7 & 30.3 & 37.9 & 8.6 \\
\hline Right wrist & 53.4 & 41.5 & 33.5 & 16.5 \\
\hline Right hand & 65.8 & 43.3 & 51.0 & 23.3 \\
\hline Left hand & 47.7 & 26.5 & 66.3 & 59.7 \\
\hline Left shoulder & 50.3 & 9.6 & 64.7 & 28.7 \\
\hline Left elbow & 44.6 & 9.9 & 62.5 & 33.1 \\
\hline Left wrist & 39.4 & 20.1 & 58.4 & 52.1 \\
\hline
\end{tabular}

* Shaded area represents the tested side.

Table 3

Mean $(\mu)$ and standard deviation $(\sigma)$ joint angle differences between Kinect and motion capture

\begin{tabular}{|c|c|c|c|c|}
\hline & \multicolumn{2}{|c|}{ Right side protocol } & \multicolumn{2}{|c|}{ Left side protocol } \\
\hline & $\mu\left(^{\circ}\right)$ & $\sigma\left(^{\circ}\right)$ & $\mu\left({ }^{\circ}\right)$ & $\sigma\left(^{\circ}\right)$ \\
\hline Right shoulder vertical angle & 19.0 & 22.4 & 24.4 & 14.4 \\
\hline Right shoulder horizontal angle & 9.03 & 6.88 & 12.0 & 4.89 \\
\hline Right elbow angle & 11.0 & 7.85 & 8.82 & 4.56 \\
\hline Left shoulder vertical angle & 20.0 & 12.3 & 18.5 & 22.0 \\
\hline Left shoulder horizontal angle & 27.9 & 4.39 & 28.3 & 7.84 \\
\hline Left elbow angle & 13.2 & 3.84 & 12.2 & 8.67 \\
\hline
\end{tabular}

* Shaded area represents the tested side.
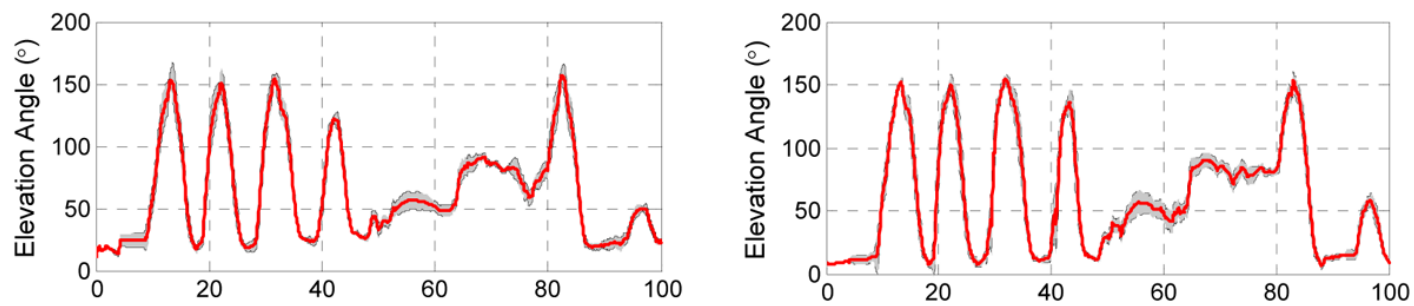

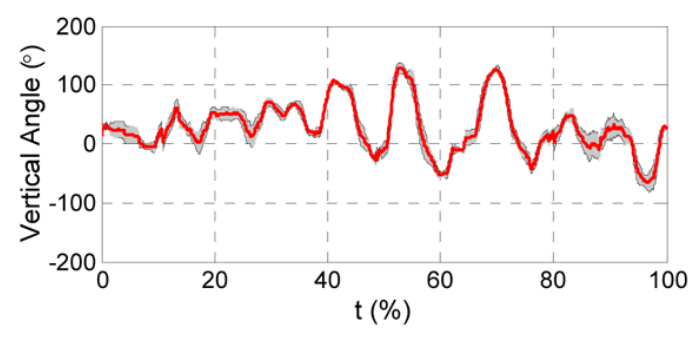

(a)

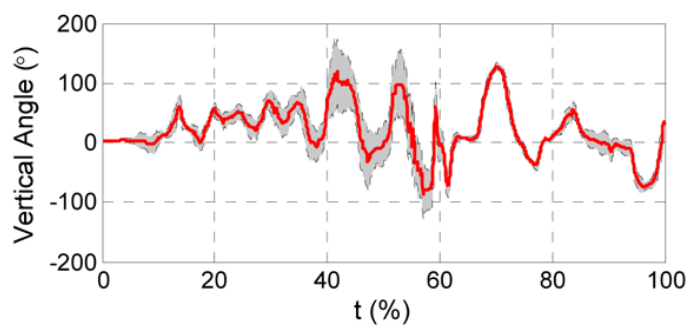

(b)

Fig. 4. The average joint angle trajectories for the elevation and rotation around the vertical axis of obtained using dynamic time warping across 10 subjects and three repetitions as captured by (a) the motion capture and (b) the Kinect. The shaded area denotes standard deviation of the samples. (Colours are visible in the online version of the article; http://dx.doi.org/ 10.3233/THC-130764)

position offsets for each subject were averaged across three trials. The mean and standard deviations of the offsets for the 10 subjects and both protocols (right and left sides) were calculated. The mean joint 
Table 4

Reachable workspace results in different groupings of the subjects

\begin{tabular}{|c|c|c|c|c|c|c|c|c|c|c|c|}
\hline \multirow[t]{3}{*}{ Group } & \multirow[t]{3}{*}{ Side } & \multicolumn{5}{|c|}{ Motion capture } & \multicolumn{5}{|c|}{ Kinect } \\
\hline & & \multicolumn{4}{|c|}{ RSA per quadrant } & \multirow[t]{2}{*}{ RSA } & \multicolumn{4}{|c|}{ RSA per quadrant } & \multirow[t]{2}{*}{ RSA } \\
\hline & & I & II & III & IV & & $\mathrm{I}$ & II & III & IV & \\
\hline $\begin{array}{c}\text { Females } \\
N=5\end{array}$ & $\begin{array}{l}\mathrm{D} \\
\mathrm{ND}\end{array}$ & $\begin{array}{r}0.122 \\
\pm 0.016 \\
0.139 \\
\pm 0.024\end{array}$ & $\begin{array}{r}0.146 \\
\pm 0.010 \\
0.180 \\
\pm 0.018\end{array}$ & $\begin{array}{r}0.193 \\
\pm 0.031 \\
0.169 \\
\pm 0.030\end{array}$ & $\begin{array}{r}0.229 \\
\pm 0.012 \\
0.224 \\
\pm 0.005\end{array}$ & $\begin{array}{r}0.690 \\
\pm 0.028 \\
0.713 \\
\pm 0.053\end{array}$ & $\begin{array}{r}0.168 \\
\pm 0.022 \\
0.180 \\
\pm 0.035\end{array}$ & $\begin{array}{r}0.151 \\
\pm 0.030 \\
0.182 \\
\pm 0.023\end{array}$ & $\begin{array}{r}0.219 \\
\pm 0.027 \\
0.206 \\
\pm 0.028\end{array}$ & $\begin{array}{r}0.232 \\
\pm 0.013 \\
0.226 \\
\pm 0.005\end{array}$ & $\begin{array}{r}0.770 \\
\pm 0.048 \\
0.794 \\
\pm 0.072\end{array}$ \\
\hline $\begin{array}{l}\text { Males } \\
N=5\end{array}$ & $\begin{array}{l}\mathrm{D} \\
\mathrm{ND}\end{array}$ & $\begin{array}{r}0.098 \\
\pm 0.040 \\
0.125 \\
\pm 0.041\end{array}$ & $\begin{array}{r}0.129 \\
\pm 0.037 \\
0.153 \\
\pm 0.034\end{array}$ & $\begin{array}{r}0.178 \\
\pm 0.013 \\
0.152 \\
\pm 0.011\end{array}$ & $\begin{array}{r}0.232 \\
\pm 0.004 \\
0.218 \\
\pm 0.004\end{array}$ & $\begin{array}{r}0.637 \\
\pm 0.083 \\
0.648 \\
\pm 0.077\end{array}$ & $\begin{array}{r}0.116 \\
\pm 0.047 \\
0.158 \\
\pm 0.057\end{array}$ & $\begin{array}{r}0.121 \\
\pm 0.034 \\
0.142 \\
\pm 0.033\end{array}$ & $\begin{array}{r}0.198 \\
\pm 0.015 \\
0.179 \\
\pm 0.014\end{array}$ & $\begin{array}{r}0.231 \\
\pm 0.003 \\
0.221 \\
\pm 0.004\end{array}$ & $\begin{array}{r}0.666 \\
\pm 0.091 \\
0.700 \\
\pm 0.094\end{array}$ \\
\hline $\begin{array}{c}\text { Combined } \\
N=10\end{array}$ & $\begin{array}{l}\mathrm{D} \\
\mathrm{ND}\end{array}$ & $\begin{array}{r}0.110 \\
\pm 0.031 \\
0.132 \\
\pm 0.033\end{array}$ & $\begin{array}{r}0.137 \\
\pm 0.027 \\
0.166 \\
\pm 0.033\end{array}$ & $\begin{array}{r}0.185 \\
\pm 0.024 \\
0.160 \\
\pm 0.023\end{array}$ & $\begin{array}{r}0.230 \\
\pm 0.009 \\
0.221 \\
\pm 0.005\end{array}$ & $\begin{array}{r}0.663 \\
\pm 0.065 \\
0.680 \\
\pm 0.071\end{array}$ & $\begin{array}{r}0.142 \\
\pm 0.044 \\
0.169 \\
\pm 0.046\end{array}$ & $\begin{array}{r}0.136 \\
\pm 0.034 \\
0.162 \\
\pm 0.034\end{array}$ & $\begin{array}{r}0.208 \\
\pm 0.023 \\
0.193 \\
\pm 0.026\end{array}$ & $\begin{array}{r}0.232 \\
\pm 0.009 \\
0.224 \\
\pm 0.005\end{array}$ & $\begin{array}{r}0.718 \\
\pm 0.088 \\
0.747 \\
\pm 0.093\end{array}$ \\
\hline $\begin{array}{c}\text { Total: } \\
N=20\end{array}$ & Both & $\begin{array}{r}0.121 \\
\pm 0.033\end{array}$ & $\begin{array}{r}0.152 \\
\pm 0.031 \\
\end{array}$ & $\begin{array}{r}0.173 \\
\pm 0.026\end{array}$ & $\begin{array}{r}0.226 \\
\pm 0.008 \\
\end{array}$ & $\begin{array}{r}0.672 \\
\pm 0.067\end{array}$ & $\begin{array}{r}0.156 \\
\pm 0.046 \\
\end{array}$ & $\begin{array}{r}0.149 \\
\pm 0.036\end{array}$ & $\begin{array}{r}0.201 \\
\pm 0.025\end{array}$ & $\begin{array}{r}0.228 \\
\pm 0.008 \\
\end{array}$ & $\begin{array}{r}0.732 \\
\pm 0.089\end{array}$ \\
\hline
\end{tabular}

*The results show mean relative surface area (RSA) and standard deviation. Refer to Fig. 2 for division of the quadrants.

position differences ranged between $33.5 \mathrm{~mm}$ and $66.3 \mathrm{~mm}$. The standard deviations ranged between $8.6 \mathrm{~mm}$ and $59.7 \mathrm{~mm}$ with the right and left hand locations having the largest standard deviations for their respective protocols. Similarly, the standard deviations were highest for all the joints of each side while subjects were performing the protocol on the same side, and they were lowest while the joints on the opposite side were held still during the protocol. The average results across all subjects are reported in Table 2.

\subsection{Angular analysis - goniometry}

The measurements of the subjects' joint angles were calculated using a similar method as described in the pose analysis. The mean differences and standard deviations for all 10 subjects are shown in Table 3. The angular differences varied greatly on both sides and throughout both protocols. The standard deviations were highest for the vertical shoulder angle, and were particularly high for each side during their respective protocol. Further analysis of the data showed that subjects 1, 4 and 7 had significantly higher standard deviations across trials which increased the overall standard deviations results.

To further examine the variability of the angular measurements, we aligned the vertical and horizontal angle trajectories obtained across 10 subjects and three repetitions using DTW. We calculated the mean and standard deviation of the aligned trajectories for the motion capture and Kinect system, respectively. The angular results of the motion capture show small but consistent variability for the motion capture across the entire movement sequence (Fig. 4(a)). On the other hand, the angular trajectories obtained from the Kinect show more variability, in particular during horizontal sweeps (Fig. 4(b)) when the arm is pointing towards the camera, resulting in less reliable pose estimation due to occlusions.

\subsection{Reachable workspace}

Figure 5 shows the RSA of the reachable workspace for non-dominant and dominant side. For each subject, we captured three repetitions whose results are shown with the triangular markers. The bars 
Relative Surface Area: Non-Dominant Side

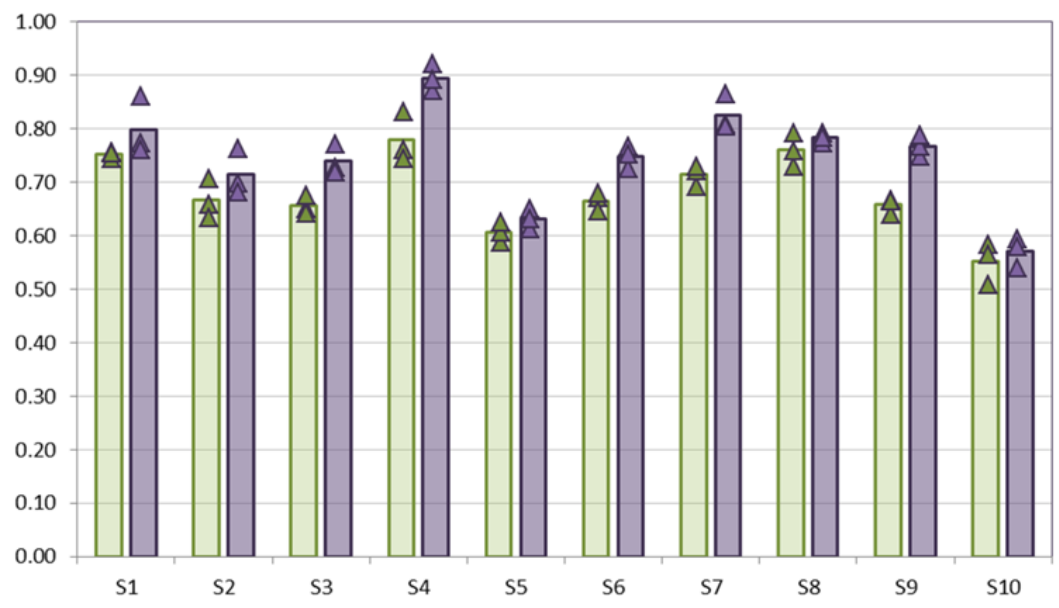

Relative Surface Area: Dominant Side

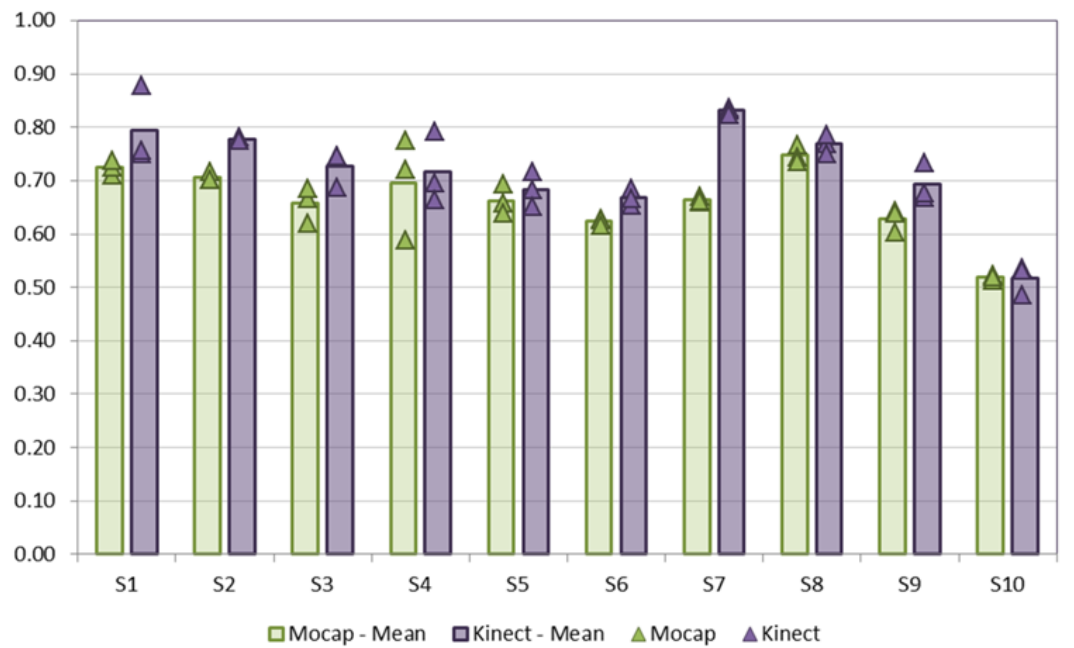

Fig. 5. Relative surface area as acquired in 10 healthy control subjects using motion capture and Kinect. The bars show mean value across the three trials denoted with triangular markers for dominant (above) and non-dominant side (below). (Colours are visible in the online version of the article; http://dx.doi.org/10.3233/THC-130764)

represent the mean values of the RSA for each subject from the motion-capture and Kinect. In most subjects, the variability between trials was low. Subjects 4, 6 and 9 exhibited considerably higher RSA in the non-dominant side while the performance for the other subjects was similar for both sides.

Two-way ANOVA was used to assess whether gender and hand dominancy had an effect on the total RSA measured by the motion capture, which was used as the reference measurement. The mean and standard deviations are presented in Table 4. The two-way ANOVA showed no significant main effect for the gender factor $\mathrm{F}(1,16)=4.23, p=0.056$; no significant main effect for the dominancy factor $\mathrm{F}(1,16)=0.36, p=0.554$; the interaction between gender and dominancy was also found to be not significant $\mathrm{F}(1,16)=0.042, p=0.840$. For further analysis, we therefore looked at combined values for gender and dominancy. Data from the Kinect showed more significant effect from gender $(P=0.012)$; but not for dominancy or combined. 


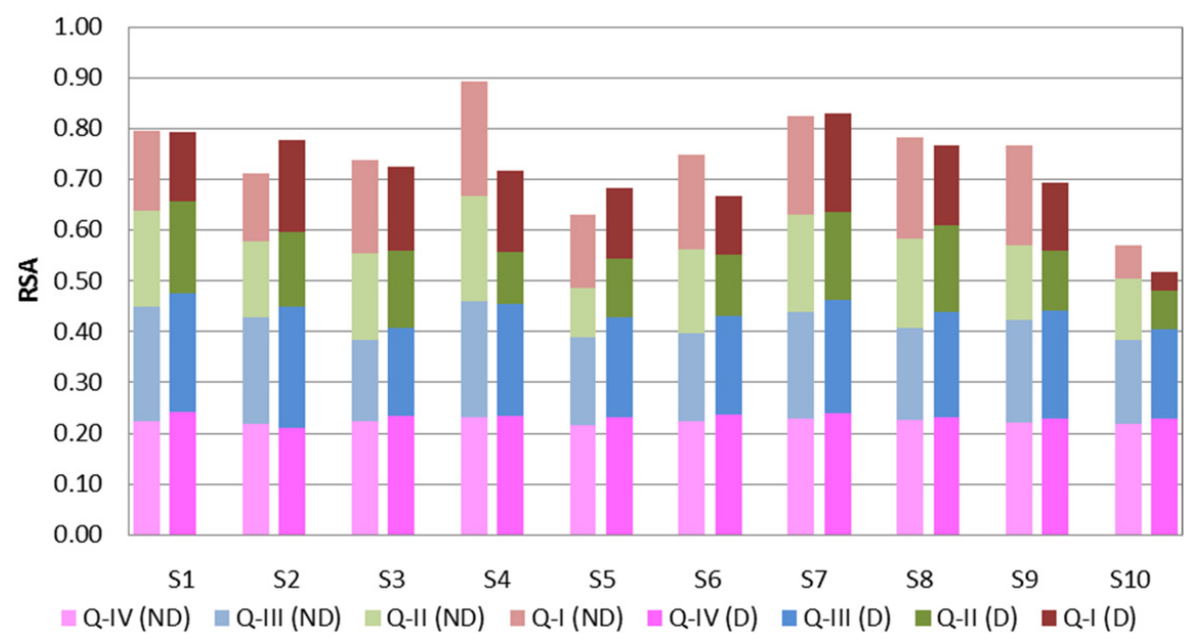

Fig. 6. Relative surface area (RSA) per quadrant (mean over three repetitions) for dominant (D) and non-dominant (ND) arm as captured by the Kinect. (Colours are visible in the online version of the article; http://dx.doi.org/10.3233/THC-130764)

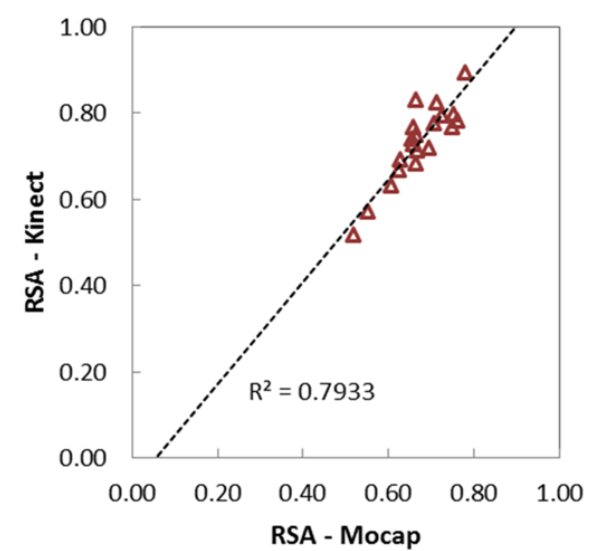

(a)

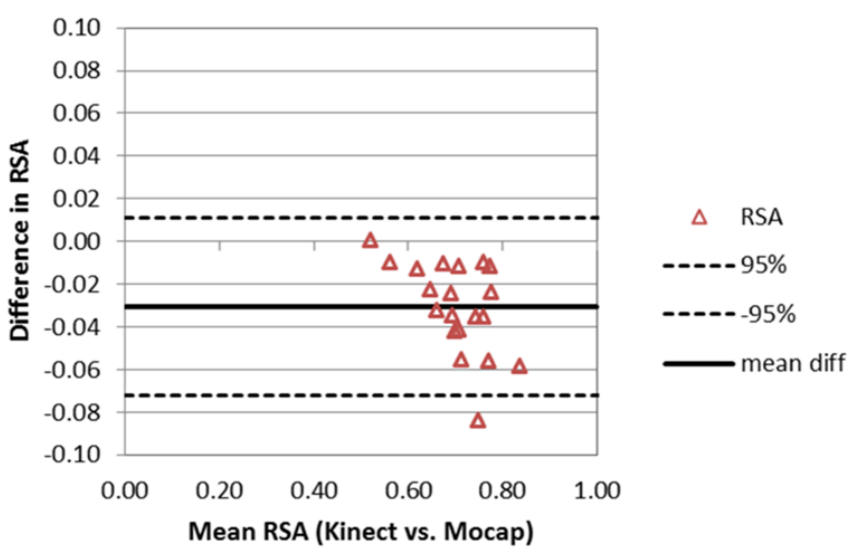

(b)

Fig. 7. (a) The total reachable workspace area as captured by the motion capture and the Kinect. (b) The corresponding Bland-Altman plot. The horizontal solid line shows the mean difference between the two systems $(-0.03)$ while the upper and lower dashed lines designate the $95 \%$ limits of agreement. (Colours are visible in the online version of the article; http://dx.doi.org/10.3233/THC-130764)

Figure 6 shows the decomposition of the assessed RSA into the four quadrants. The results show high consistency in the quadrants III and IV with more variability in the two contralateral quadrants I and II (those that require reaching across the body).

\subsubsection{Validity}

Figure 7(a) shows the scatter plot of the total reachable surface area (RSA) obtained by the two systems. The analysis shows high correlation between the datasets $\left(R^{2}=0.79, \mathrm{~F}(1,19)=69.07, p<\right.$ 0.001). In almost all the subjects, measurement with the Kinect resulted in slightly larger RSA values. Figure 7(b) shows the corresponding Bland-Altman plot. The majority of the measurements lie within the $95 \%$ agreement intervals of the mean difference $(-0.03)$. The offset suggests that the Kinect measurements somewhat overestimate the movement trajectory. Furthermore, a paired-samples t-test was 


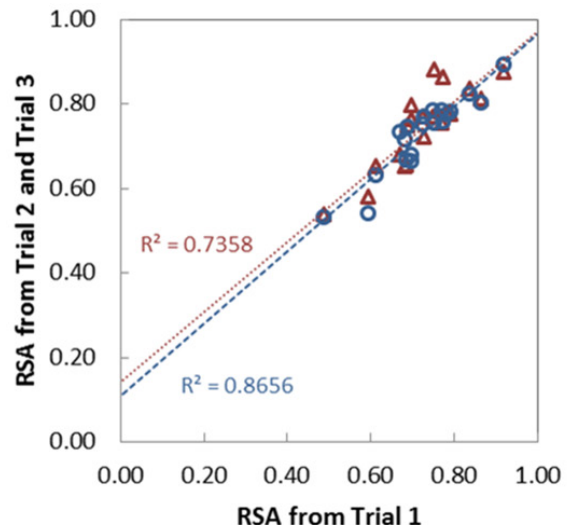

(a)

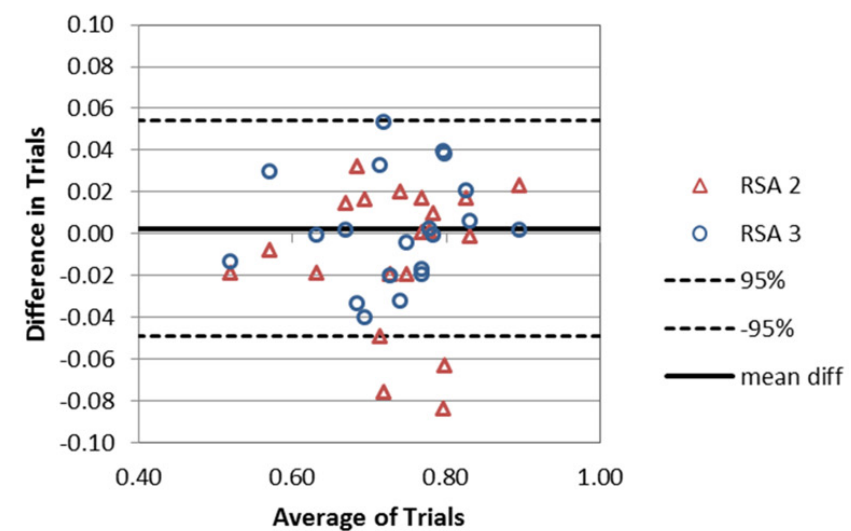

(b)

Fig. 8. (a) Reproducibility of the total reachable workspace area at trial 2 and trial 3 versus trial 1. (b) The Bland-Altman plot for the total reachable surface area. The mean difference was 0.0024 . The upper lines and lower lines designate the $95 \%$ limits of agreement. (Colours are visible in the online version of the article; http://dx.doi.org/10.3233/THC-130764)

conducted to compare the total RSA captured by the two systems in individual subjects. There was no significant difference in the total RSA for motion capture $(\mathrm{M}=0.672, \mathrm{SD}=0.067)$ and Kinect $(\mathrm{M}=$ $0.732, \mathrm{SD}=0.089) ; t(19)=-6.38, P<0.0001$.

\subsubsection{Reliability}

To assess the test-retest reliability of the Kinect-based assessment, we analyzed the correlation between the first and second trials and the first and third trials. Figure 8(a) shows the reproducibility of the total reachable workspace area during trial 2 and trial 3 compared to trial 1 . The results show high agreement between the trials with correlation coefficients of 0.86 and 0.93 respectively (trial 2 vs. trial 1: $R^{2}=0.74, \mathrm{~F}(1,19)=50.13, p<0.001$; trial 3 vs. trial $\left.1: R^{2}=0.87, \mathrm{~F}(1,19)=115.9, p<0.001\right)$. The Bland-Altman plot in Fig. 8(b) shows the dispersion of the individual reliability of the total RSA. The mean difference in total RSA was small (0.0024) and not significantly different from zero, with the majority of the data points within the $95 \%$ limits of agreement.

\section{Discussion}

In addressing the clinical needs for an outcome measure for upper-limb function, our goal was to develop a methodology that would be reliable, easy to administer, time-efficient, and cost-effective, while being able to capture an individual's impairments related to functional space of the upper limbs. To address the validity and reliability of the proposed methodology, we performed a three-step evaluation by examining (1) joint positions, (2) joint angles, and (3) reachable workspace computations.

The joint position offsets and their standard deviations were the highest for all the joints of each side while subjects were performing their respective protocols. The offsets had the lowest values while the joints on the opposite side were held still during the protocol. These findings were expected since the joint positions are most accurately measured when the subject is not moving. It should be noted that subject 4 had the highest variability across trials, thus potentially skewing the results of both the joint position and joint angle offsets. Our findings suggest that the Kinect is accurate within $66.3 \mathrm{~mm}$ 


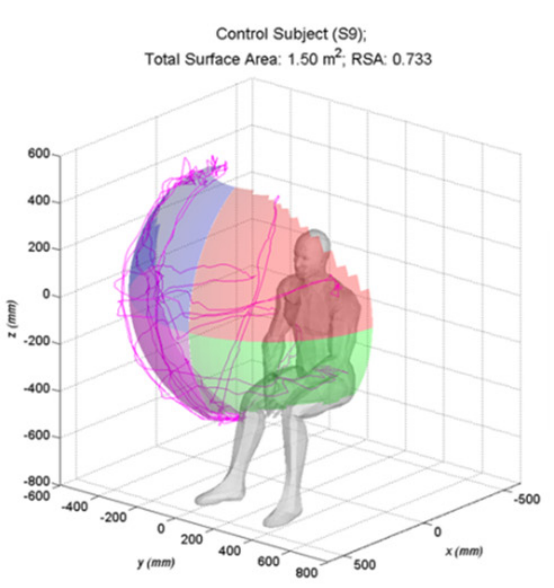

(a)
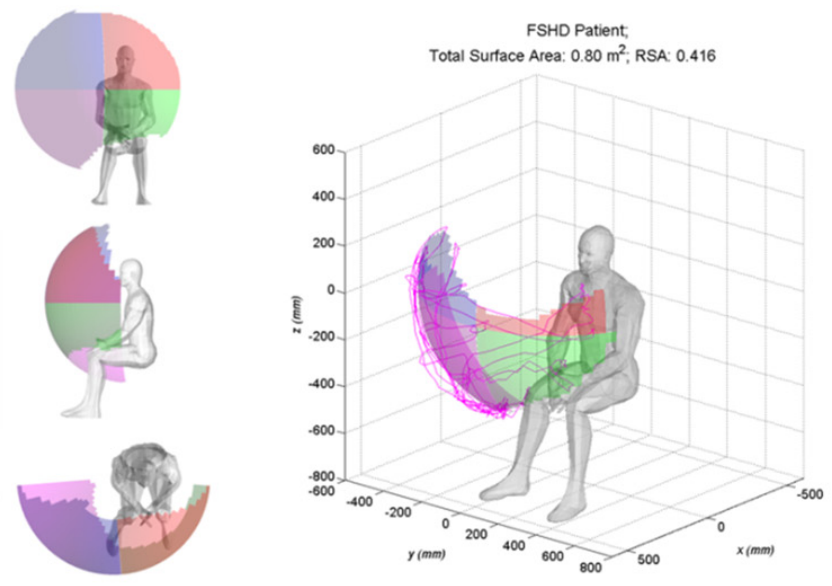

(b)

Fig. 9. 3D view and planar projections of the reachable workspace acquired in a healthy control subject (S9) and patient with FSHD. Note the reduction of the workspace in the upper quadrants of the patient's data due to shoulder weakness which is typical for this form of neuromuscular disease. (Colours are visible in the online version of the article; http://dx.doi.org/ 10.3233/THC-130764)

compared to the motion-capture system for our experimental protocol. As previously stated, these offsets arise from the differences in the technologies and inherent kinematic models of the individual systems.

The measurement of the joint angle offsets between the two systems proved to be more difficult to accurately quantify. Although the key poses of our movement protocol are similar to those used in the active ROM measurement, the peak angle measurements cannot be directly compared due to the dynamic nature of the motion capture and Kinect systems. As previously noted, subjects 1, 4 and 7 had the highest standard deviations for the measurements of joint angles. These subjects had the highest active ROM goniometric measurements and were all female (Table 1). The effect of gender on the accuracy is later discussed.

To minimize additional transient errors due to differences in system performance, DTW was also used to calculate both the joint position and angle offsets. The differences in the results were minimal for the joint position offsets $(<2 \%)$ while the differences in the joint angle offsets were typically $<10 \%$. This suggested that the system performance was likely not a significant factor in error contributions. Additionally, the analyses of the data did not vary based on the results of the DTW data.

The analysis of variance for the effects of gender and hand dominancy on the total RSA values showed no significant effects of the two parameters. The Kinect data, however, showed significant effect of gender. In our previous study [19] on a larger population $(N=20)$, we found no effect of gender on the reachable workspace performance. The groups in this study were considerably smaller while being more diverse with respect to age. The female group of this study included two subjects, both 17 years old, who scored high values. We hypothesize that by examining the reachable workspace of a larger group of female and male subjects of different age groups, some differences would be revealed in total RSA. In particular, the upper quadrants would most likely vary since they require a higher level of flexibility. Other studies of upper limb ROM, which is closely related to reachable workspace, reported the effect of gender to be much weaker than that of age [32]. Similarly, the effect of hand dominancy on ROM was found to be significant for some of the joints, but the reported difference were small and thus not clinically relevant [1]. 
We also examined the validity of the Kinect-based assessment with respect to the motion-capture. While high agreement between the two systems was found, the Kinect measurements somewhat overestimated the movement trajectories and consequently the corresponding RSA values. The skeletal tracking on the Kinect includes several filters which are adjustable for various applications that trade-off between accurate tracking and smoothness of movement trajectories. Our joint filtering parameters were set as follows: 0.5 for smoothing, 0.5 for correction, 0.5 for prediction, $5.0 \mathrm{~cm}$ for jitter radius, and $4 \mathrm{~cm}$ for maximum deviation radius (as defined by Kinect SDK), which produce smoother trajectories with small latency. While the low latency faithfully tracked the actual joint positions, the predictive filter still generated some overshooting when faster movements were executed or movement direction was altered.

Finally, we investigated the reliability of the reachable workspace measurement by performing testretest analysis of three consecutive trials. The Bland-Altman analysis in conjunction with the correlation between 0.86 and 0.93 suggested a high repeatability between trials. We believe that the visual feedback assisted the subjects during the assessment to help them correctly perform the movement protocol. Future reliability analyses over a course of several days are needed to evaluate the effect of biological variability on the proposed measure.

To illustrate the application of this methodology as a potential outcome measure of upper limb function, we show the difference in the reachable workspace between a healthy control (left) and a subject with FSHD (right) in Fig. 9. One of the typical symptoms of FSHD is shoulder girdle muscle weakness as exhibited in difficulty raising hand overhead. The results in Fig. 9(b) show considerable reduction in the reachable workspace of the upper two quadrants, while the functionality in the lower two quadrants are well preserved and similar to that of the healthy control. With a clinical test using Brooke upper extremity functional scale [5], the subject's functional grade for the upper extremity was 3 , which is explained as an inability to "raise hands above head but can raise an $8 \mathrm{oz}$. glass/cup of water to mouth (using both hands if necessary)". The graphical representation of the patient's reachable workspace with its deficient areas in the upper quadrant correlates well with the functional status of the patient.

One of the limitations of this evaluation study was the small sample size of subject groups with respect to gender and age. We chose to include subjects of wide age range to evaluate the applicability of the developed system in general population. Additionally, the kinematic model of the reference skeleton was simplified to assume an ideally spherical workspace. Although the kinematic structure of the shoulder joint is much more complex $[14,18]$ than presented in this paper, we attempted to balance the tradeoffs between complexity and accuracy with versatility and clinical relevancy of the developed outcome measures. We hypothesize that a more elaborate model would not considerably improve the outcomes of the method because the biological variability of the movement is greater than the accuracy gained from using such a highly accurate model. Future studies may examine modifications to the kinematic model for different clinical or research applications.

\section{Conclusions}

In this study, we examined the validity and reliability of a novel method for assessing upper-limb workspace using the Microsoft Kinect system. The findings indicate that the proposed 3D reachable workspace analysis provides sufficiently accurate and reliable results as compared to motion-capture system. The analysis of joint positions and angles showed that the dynamic measurements had large deviations in certain poses (e.g. arm extending towards the camera) and particularly with some female subjects. To improve the accuracy of the angular measurements, the output of the Kinect tracking could be calibrated with respect to more accurate motion capture and corrected for using regression equations 
as suggested in [33]. Despite relatively large differences in average angle and joint positions, we showed that the reachable workspace envelope as proposed in this study was robust to such deviations.

The results of this study indicate that the proposed methodology could be valuable for clinical evaluation of upper extremity in neurological or musculoskeletal conditions. The RSA of the workspace could provide more sensitive and useful global upper extremity functional measure for following disease progression or effects of various therapeutic interventions. In addition, the obtained reachable workspace data can be further correlated with patient-reported functional deficits in activities of daily living (ADL) or different standardized and established clinical measures. In future studies, evaluation of the sensitivity of the system in longitudinal studies and correlation with clinically-meaningful upper extremity functional status will have additional prognostic implications for this novel outcome measure.

\section{Acknowledgements}

The research was supported by Center for Information Technology Research in the Interest of Society (CITRIS) at University of California, Berkeley; National Science Foundation (NSF): \#1111965; and U.S. Department of Education/NIDRR: \#H133B031118 and \#H133B090001; Parent Project Muscular Dystrophy (PPMD): Exploratory Grant. We thank Alina Nicorici and Linda Johnson of UC Davis Medical Center for assistance with the experimental protocols.

\section{References}

[1] Macedo LG, Magee DJ. Differences in range of motion between dominant and nondominant sides of upper and lower extremities. J Manipulative Physiol Ther. 2008; 31(8): 577-582.

[2] Sharpless J. The nine hole peg test of finger hand coordination for the hemiplegic patient. In: Sharpless J. A Problem Oriented Approach to Stroke Rehabilitation. Springfield: Charles C. Thomas; 1982. p. 470-473.

[3] Jebsen RH, Taylor N, Trieschmann RB, Trotter MJ, Howard LA. An objective and standardized test of hand function. Arch Phys Med Rehabil. 1969; 50(6): p. 311-319.

[4] Wolf SL, Catlin PA, Ellis M, Archer A, Morgan B, Piacentino A. Assessing Wolf Motor Function Test as outcome measure for research in patients after stroke. Stroke. 2001; 32(7): 1635-1639.

[5] Brooke M, Griggs R, Mendell J, Fenichel G, Shumate J, Pellegrino R. Clinical trial in Duchenne dystrophy. I. The design of the protocol. Muscle Nerve. 1981; 4(3): 186-197.

[6] Lang C, Bland M, Bailey R, Schaefer S, Birkenmeier R. Assessment of upper extremity impairment, function, and activity after stroke: foundations for clinical decision making. J Hand Ther. 2013; 26(2): 104-114.

[7] Mazzone E, Vasco G, Palermo C, Bianco F, Galluccio C, Ricotti V, Castronovo AD, Mauro MS, Pane M, Mayhew A, Mercuri E. A critical review of functional assessment tools for upper limbs in Duchenne muscular dystrophy. Dev Med Child Neurol. 2012; 54(10): 879-885.

[8] Roller A, Mounts R, DeLong J, Hanypsiak B. Outcome instruments for the shoulder. Arthroscopy. 2013; 29(5): 955-964.

[9] Hudak P, Amadio P, Bombardier C. Development of an upper extremity outcome measure: The DASH (disabilities of the arm, shoulder and hand). Corrected. Am J Ind Med. 1996; 29(6): 602-608.

[10] Mullaney M, McHugh M, Johnson C, Tyler T. Reliability of shoulder range of motion comparing a goniometer to a digital level. Physiother Theory Pract. 2010; 26(5): 327-333.

[11] Zacharias F, Borst C, Hirzinger G. Capturing robot workspace structure: representing robot capabilities. In: Proceedings of IEEE/RSJ International Conference on Intelligent Robots and Systems (IROS); 2007. p. 3229-3236.

[12] Kennedy K. Reach capability of men and women: A three dimensional analysis. Report No. AMRL-tr-77-50. OH: Aerospace Medical Research Laboratories; 1978.

[13] Li S, Xi Z. The measurement of functional arm reach envelopes for young Chinese males. Ergonomics. 1999; 33: 967978.

[14] Abdel-Malek K, Yang J, Brand R, Tanbour E. Towards understanding the workspace of human limbs. Ergonomics. 2004; 47: 1386-1405.

[15] Behara D, Das B. Anthropometric modelling for the determination of 3D maximum functional reach. Theoretical Issues in Ergonomics Science. 2010; 12: 87-101. 
[16] Zacharias F, Howard IS, Hulin T, Hirzinger G. Workspace comparisons of setup configurations for human-robot interaction. In: Proceedings of IEEE/RSJ International Conference on Intelligent Robots and Systems (IROS); 2010; Taipei, Taiwan.

[17] Schiele A, Helm FCT, Kinematic design to improve ergonomics in human machine interaction. IEEE Trans Neural Syst Rehab Eng. 2006; 14(4): 456-469.

[18] Klopcar N, Lenarcic J. Kinematic model for determination of human arm reachable workspace. Meccanica. 2005; 40: 203-219.

[19] Kurillo G, Han JJ, Abresch RT, Nicorici A, Yan P, Bajcsy R. Development and application of stereo camera-based upper extremity workspace evaluation in patients with neuromuscular diseases. PLoS ONE. 2012; 7(9).

[20] Zhang Z. Microsoft Kinect sensor and its effect. IEEE MultiMedia. 2012; 19(2): 4-12.

[21] Clark RA, Pua YH, Fortin K, Ritchie C, Webster KE, Denehy L, Bryant AL. Validity of the Microsoft Kinect for assessment of postural control. Gait and Posture. 2012; 36: 372-377.

[22] Stone E, Skubic M. Evaluation of an inexpensive depth camera for in-home gait assessment. Journal of Ambient Intelligence and Smart Environments. 2011; 3(4): 349-361.

[23] Lowes L, Alfano L, Yetter B, Worthen-Chaudhari L, Hinchman W, Savage J, Samona P, Flanigan KM, Mendell JR. Proof of concept of the ability of the Kinect to quantify upper extremity function in dystrophinopathy. PLoS Currents. 2013; 14(5).

[24] Chang CY, Lange B, Zhang M, Koenig S, Requejo P, Somboon N, Sawchuk A, Rizzo AA. Towards pervasive physical rehabilitation using Microsoft Kinect. In Proceedings of PervasiveHealth; 2012. p. 159-162.

[25] Fernandez-Baena A, Susın A, Lligadas X. Biomechanical validation of upper-body and lower-body joint movements of Kinect motion capture data for rehabilitation treatments. In: Proceedings of $4^{\text {th }}$ International Conference on Intelligent Networking and Collaborative Systems; 2012. p. 656-661.

[26] Choppin S, Wheat J. The potential of the Microsoft Kinect in sports analysis and biomechanics. Sports Technology. 2013; 6(2): 78-85

[27] Obdržálek Š, Kurillo G, Ofli F, Bajcsy R, Seto E, Jimison H, Pavel M. Accuracy and robustness of Kinect pose estimation in the context of coaching of elderly population. In Proceedings of $34^{\text {th }}$ International Conference of the IEEE Engineering in Medicine and Biology Society (EMBC); 2012; San Diego, CA.

[28] Kurillo G, Han JJ, Obdržálek Š, Yan P, Abresh RT, Nicorici A, Yan P, Bajcsy R. Upper extremity reachable workspace evaluation with Kinect. Stud Health Technol Inform. 2013; 184: 247-253.

[29] Khoshelham K, Elberink SO. Accuracy and resolution of Kinect depth data for indoor mapping applications. Sensors. 2012; 12(2): 1437-1454.

[30] Edelsbrunner H, Mücke EP. Three-dimensional alpha shapes. In: Proceedings of the 1992 workshop on Volume visualization; 1992. p. 75-82.

[31] Catmull E, Rom R. A class of local interpolating splines. In: Barnhill RE, Riesenfled RF, editors. Computer Aided Geometric Design. New York: Academic Press; 1974. p. 317-326.

[32] Doriot N, Wang X. Effects of age and gender on maximum voluntary range of motion of the upper body joints. Ergonomics. 2006; 49(3): 269-281.

[33] Bonnechère B, Jansen B, Salvia P, Bouzahouene H, Omelina L, Cornelis J, Rooze M, Van Sint S. What are the current limits of the Kinect ${ }^{\mathrm{TM}}$ sensor? In: Proceedings of 9th International Conference on Disability, Virtual Reality and Associated Technologies; 2012; Laval, France. p. 287-294. 